\title{
Targeting the autosomal Ceratitis capitata transformer gene using Cas9 or dCas9 to masculinize $\mathrm{XX}$ individuals without inducing mutations
}

Pasquale Primo ${ }^{1 \dagger}$, Angela Meccariello ${ }^{1 \dagger}$, Maria Grazia Inghilterra ${ }^{1}$, Andrea Gravina', Giuseppe Del Corsano ${ }^{1}$, Gennaro Volpe ${ }^{1}$, Germano Sollazzo ${ }^{1}$, Serena Aceto ${ }^{1}$, Mark D. Robinson², Marco Salvemini ${ }^{1}$ and Giuseppe Saccone ${ }^{1 *}$

\begin{abstract}
Background: Females of the Mediterranean fruit fly Ceratitis capitata (Medfly) are major agricultural pests, as they lay eggs into the fruit crops of hundreds of plant species. In Medfly, female sex determination is based on the activation of Cctransformer (Cctra). A maternal contribution of Cctra is required to activate Cctra itself in the XX embryos and to start and epigenetically maintain a Cctra positive feedback loop, by female-specific alternative splicing, leading to female development. In XY embryos, the male determining Maleness-on-the-Y gene (MoY) blocks this activation and Cctra produces male-specific transcripts encoding truncated CcTRA isoforms and male differentiation occurs.

Results: With the aim of inducing frameshift mutations in the first coding exon to disrupt both female-specific and shorter male-specific CcTRA open reading frames (ORF), we injected Cas9 ribonucleoproteins (Cas9 and single guide RNA, sgRNA) in embryos. As this approach leads to mostly monoallelic mutations, masculinization was expected only in $G_{1} X X$ individuals carrying biallelic mutations, following crosses of $G_{0}$ injected individuals. Surprisingly, these injections into XX-only embryos led to $G_{0}$ adults that included not only XX females but also $50 \%$ of reverted fertile $X X$ males. The $G_{0} X X$ males expressed male-specific Cctra transcripts, suggesting full masculinization. Interestingly, out of six $G_{0} X X$ males, four displayed the Cctra wild type sequence. This finding suggests that masculinization by Cas9-sgRNA injections was independent from its mutagenic activity. In line with this observation, embryonic targeting of Cctra in XX embryos by a dead Cas9 (enzymatically inactive, dCas9) also favoured a male-specific splicing of Cctra, in both embryos and adults.

Conclusions: Our data suggest that the establishment of Cctra female-specific autoregulation during the early embryogenesis has been repressed in XX embryos by the transient binding of the Cas9-sgRNA on the first exon of the Cctra gene. This hypothesis is supported by the observation that the shift of Cctra splicing from female to male mode is induced also by dCas9. Collectively, the present findings corroborate the idea that a transient embryonic inactivation of Cctra is sufficient for male sex determination.
\end{abstract}

Keywords: iCRISPR, Sex determination, Ceratitis capitata, Epigenetics, Autoregulation, Transformer

\footnotetext{
* Correspondence: giuseppe.saccone@unina.it

${ }^{\dagger}$ Pasquale Primo and Angela Meccariello contributed equally to this work. 'Department of Biology, University of Naples Federico II, 80126 Naples, Italy Full list of author information is available at the end of the article
} 


\section{Background}

In the last few decades, the Mediterranean fruit fly Ceratitis capitata (Tephritidae, Medfly) has become a major invasive agricultural pest worldwide, following its spread from Africa and its globalization [1]. For the local suppression of this invasive species, alternatives to the use of pesticides are genetic control strategies. One of them is the Sterile Insect Technique (SIT), which has been applied successfully over the last six decades in various countries [2].

The prerequisites of SIT include a method to mass rear the target species in a cost-effective way and a method to sterilize them with a low impact on their fitness once released. As the released sterile females contribute to the fruit crop mechanical damage with the ovipositor and consequent infections, and the sterile males tend to mate with the released females rather than with the wild ones, it is highly preferable to develop a method of sexing and only release sterile males [3]. A number of strategies have been developed, including transgenic approaches for sexing, which allow the mass rearing of the two sexes, and sorting the males at the expanded last generation before the release. These strains can be based on the expression of a conditional femalelethal dominant gene [4] or on the transformation of genotypic female individuals into males by manipulating a gene involved in female sex determination [5]. Molecular genetics studies on Medfly sex determination have been useful for this aim, uncovering a cascade of regulatory genes widely conserved in the Tephritidae family (Fig. 1) [6-10]. This taxon includes many other invasive agricultural pests, such as species of the Bactrocera and Anastrepha genera [11, 12]. This fundamental knowledge is not only interesting and valuable per se [13], but also useful to develop novel sexing strategies necessary to improve the applicability of SIT. Evolutionary conservation of homologous genes and the use of transgenesis and/or CRISPR/Cas9 potentially will enable the realization of additional versatile sexing methods that can be applied in different species [8, 9, 14-16].

The sex determination of Medfly is based, as in Drosophila melanogaster, on sex-specific alternative splicing

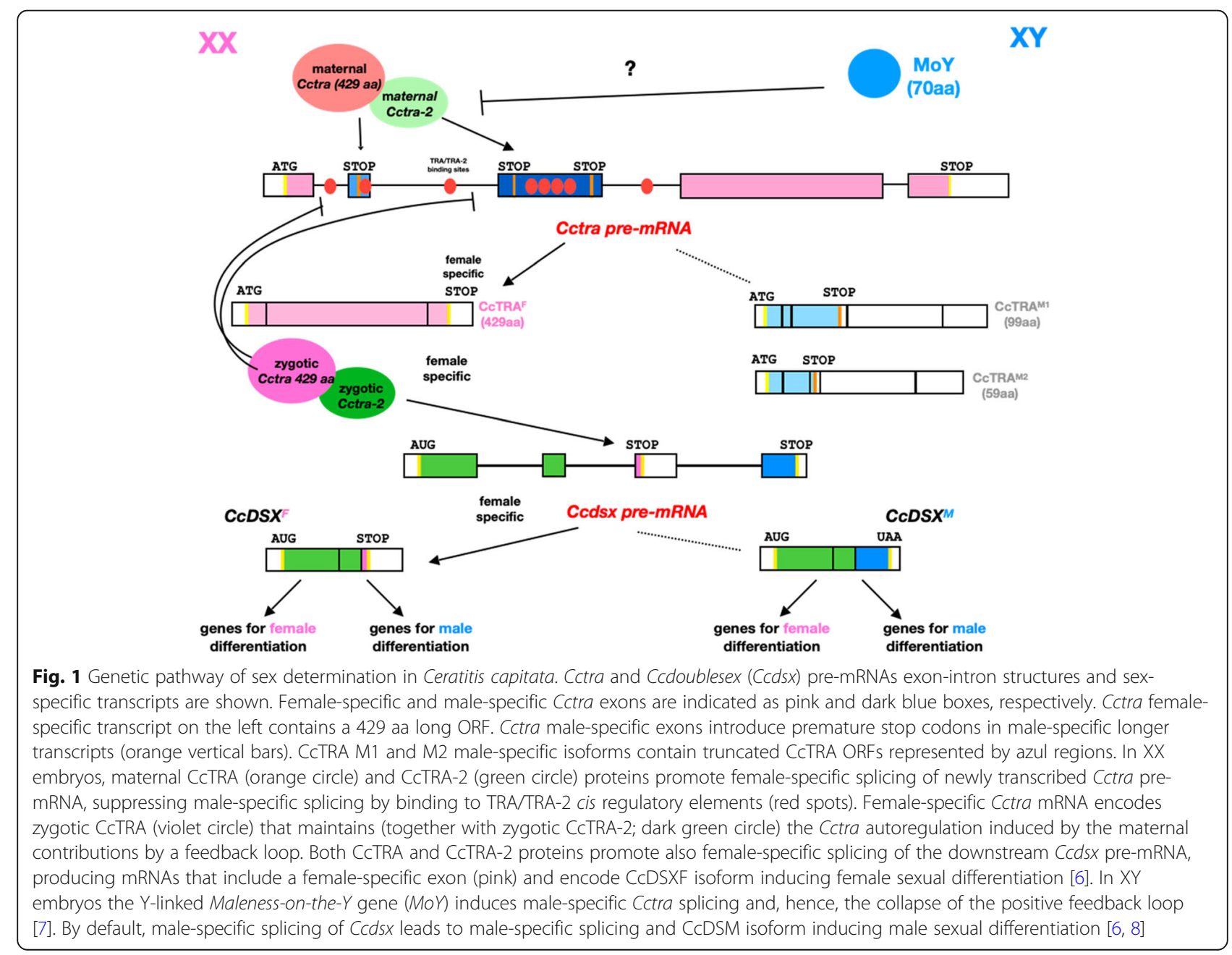


of key regulatory genes, including transformer (tra), transformer-2 (tra-2) and doublesex (dsx) orthologues (Fig. 1) [6, 7, 17-20]. Cctra is a sex determining genetic switch, which is set to $\mathrm{ON}$ in $\mathrm{XX}$ embryos and to OFF in $\mathrm{XY}$ embryos during a narrow temporal window at 5$6 \mathrm{~h}$ from oviposition [8]. In contrast to Drosophila, in XX Medfly embryos, Cctra and the auxiliary Cctransformer-2 (Cctra-2) maternal mRNAs are also required to establish a stable activation of Cctra by female-specific splicing which relies on a positive feedback loop $[6,19]$. The female-specific Cctra mRNA encodes a full-length 429 aa protein arising from the translation of an evolutionarily conserved ORF contained in the first, fourth and fifth exons (Fig. 1). In males, two longer alternative Cctra RNA isoforms, containing all five exons, encode for two truncated CcTRA proteins called CcTRA M1 (59 aa) and CcTRA M2 (99 aa) (Fig. 1). Embryonic transient RNA interference targeting mRNAs of either genes led to XX males, which are fertile even in the absence of the Y chromosome [6, 19]. As in Drosophila, Cctra and Cctra-2 are required for the female-specific splicing of Ccdoublesex $(C c d s x)$. The observation that some XX individuals are transformed in gynandromorphs (showing male-specific antennae and ovipositor or no antennae and male gonads) suggests that sex determination is cell-autonomous, as in Drosophila [6]. A TRA/TRA-2 binding element (13 nt long) is present in multiple copies in the $C c d s x$ female-specific exon, permitting a positive regulation by CCTRA/CcTRA-2, which leads to the use of this exon in $C c d s x$ transcripts of $\mathrm{XX}$ individuals, similarly to Drosophila $[7,18]$. In contrast to Drosophila, multiple copies of this splicing regulatory element are also present in Cctra locus, within and in proximity to the malespecific exons. In this other novel case, these cis elements mediate, by CcTRA/CcTRA-2 binding, exon skipping in $\mathrm{XX}$ individuals leading to CcTRA-encoding femalespecific mRNAs [6]. In XY embryos, Maleness-on-the- $Y$ $(M o Y)$ encodes a novel short protein, MOY, of still unknown biochemical function, that leads, either directly or indirectly, to male-specific splicing of Cctra and exons inclusion, at 5-6h from oviposition [8]. The presence of male-specific exons introducing stop codons in the 429 aa long Cctra ORF leads to two major RNAs encoding truncated polypeptides (respectively 59 and 99 aa long) and hence considered to be non-functional [6].

We planned to experimentally confirm this deduction, by Cas9-induced mutations in the Cctra, which would impact both the male- and female-specific ORFs. Furthermore, the availability of an efficient single RNA to induce mutations in a female-determining gene would open the future possibility to develop a gene drive strategy aimed at manipulating the sex ratio and hence the reproduction rate of this harmful species [4]. CRISPR/Cas9 has been used in the Medfly genome to target autosomal genes having two copies for each cell [16, 21, 22]. After targeting the white eye Medfly gene by injecting into early embryos in vitro pre-assembled and solubilized Cas9 ribonucleoprotein complexes (RNPs), containing sgRNA, adults showed partial mutant phenotypes caused by somatic mosaicism [16]. The most extreme mutant phenotype consisted of a fly mosaic with one of the two eyes fully colorless. While biallelic mutations were observed only in somatic clones of the fly, the germ line transmission rate was very high, reaching $100 \%$ in one case. On the contrary, targeting a Medfly single copy gene, as the Y-linked $M o Y$, in XY individuals by Cas9 ribonucleoproteins injections, $70 \%$ of mutant $G_{0}$ individuals showed intersexual phenotype and $30 \%$ were transformed into XY mutant $G_{0}$ females [8].

We reasoned that introducing loss-of-function frameshift mutations in the first Cctra exon with CRISPR/ Cas9 would lead to mutant alleles coding for truncated CcTRA proteins. The expected truncations would affect not only the 429 aa long female-specific ORF, but also the carboxy-terminal ends of two male-specific 59 and 99 aa ORFs. A masculinization of XX individuals by permanent loss-of-function mutations of Cctra altering also the male-specific CcTRA polypeptides would support the previous suggestion that these products are indeed non-functional.

Since in Drosophila tra and tra-2 mutant alleles are recessive, we reasoned that also in the Medfly the presence in the same cell and in its clonal descendants of only monoallelic indel (insertion/deletion) mutations in Cctra (+/- heterozygous state) would be insufficient to masculinize XX cells. Thus, in the somatic mutant clones of these XX individuals, the CcTRA protein expressed from the wild-type allele would lead to female-specific splicing of Cctra pre-mRNAs from both wild-type and mutant alleles, leading to female sexual phenotype. Hence, only biallelic loss-of-function Cctra mutations (Fig. 2; Cctra ${ }^{1-} / \mathrm{Cctra}^{2-}$ ) in the same XX cell and its cellular descendants would lead to a malespecific Cctra and the downstream Ccdsx RNAs, causing a partial (mosaicism) or full (very early and high biallelic mutagenesis) masculinization of XX adults.

\section{Results}

\section{Cas9-RNP injections targeting Cctra lead to fully} masculinized $X X$ flies in the $G_{0}$ progeny

With the aim of inducing indels leading to frameshift mutations in Cctra, we used Cas9 RNPs injections as a delivery method. In particular, a CRISPR/Cas9 Cctra target site was chosen within the coding region of exon 1 on the antisense strand to design a single guide RNA, named sgtraEx1 (Additional file 1: Fig. S1A). The targeted $20 \mathrm{bp}$ long sequence is about $20 \mathrm{bp}$ upstream of the first donor splicing site and upstream to the female- 


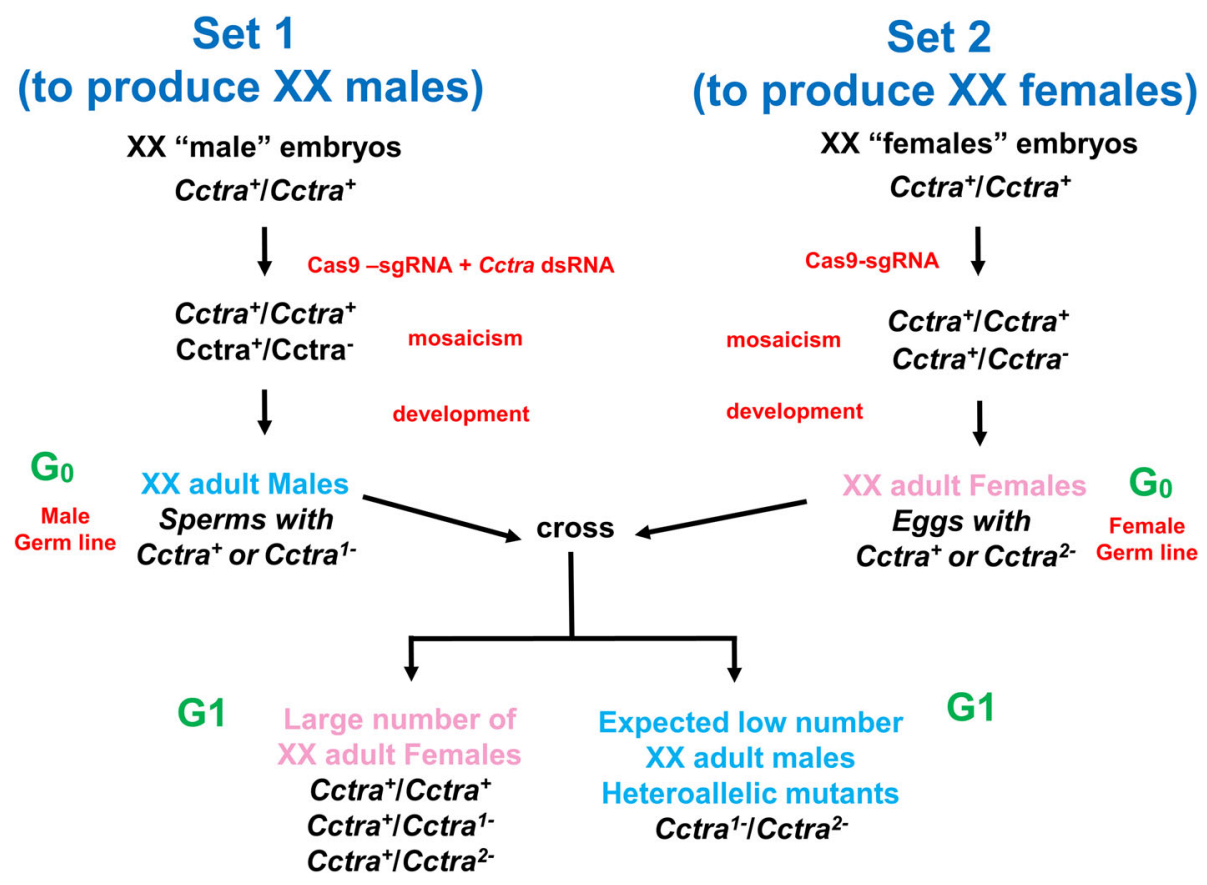

Fig. 2 Experimental design to produce $G_{1} X X$ males bearing heteroallelic mutation of Cctra. Two sets of parallel Cas9-RNP injections to target Cctra into XX embryos can lead to both $G_{0} X X$ males and XX females. The $G_{0}$ adults from the two sets can bear some Cctra monoallelic independent mutations (mosaicism) both in the soma and in the germ line. In set $\mathrm{n}$. 1, the injection mix contains also Cctra-dsRNA to induce full masculinization of $X X$ embryos (Pane et al., 2002). Hence, the $G_{0} X X$ males from set $n$. 1 can be crossed with $G_{0} X X$ females from set $n$. 2, to produce a $G_{1} X X$ progeny, expected to be composed mostly of $X X$ females (carrying either none or monoallelic mutations) and of few $G_{1} X X$ males, carrying heteroallelic mutations of $\mathrm{Cctra}\left(\mathrm{Cctra}^{1-} / \mathrm{Cctra}^{2-}\right)$ inherited from the respective parents

specific long open reading frame encoding the CcTRA protein (Additional file 1: Fig. S1B). As described above, previous literature data showed that Cas9 RNPs applied to Medfly embryos led to a relatively low somatic biallelic mutation rate overall but a high rate in the germ line [16]. Based on this study, we speculated to observe masculinization of XX Cctra mutant individuals only at $G_{1}$ after crossing $G_{0} X Y$ males and $G_{0} X X$ females developed from Cas9-injected. However, the discrimination of rare $\mathrm{Cctra}^{1-} / \mathrm{Cctra}^{2-}$ heteroallelic mutant $\mathrm{XX}$ males among the $50 \%$ of the $\mathrm{G}_{1}$ progeny being $\mathrm{XY}$ males would have been tedious and challenging. We simplified their identification by planning to detect them among a $G_{1}$ female-only $\mathrm{XX}$ progeny, obtained crossing $\mathrm{XX}$ males and females obtained from RNP-injected embryos (Fig. 2). Among these $\mathrm{G}_{1} \mathrm{XX}$ females, $\mathrm{Cctra}^{-1-} / \mathrm{Cctra}^{2-} \mathrm{XX}$ males would be easily detected even if very few, with the respect of the majority of XX females carrying either none or only one Cctra mutant allele (Fig. 2).

To produce a larger number of XX males (Fig. 2, set 1), from Cas9-injected XX embryos, a mix of Cas9-sgtraEx1 RNP and Cctra dsRNA, to efficiently masculinize XX, was injected into $370 \mathrm{XX}$ embryos (Table 1, set n. 1). The aim was to obtain XX fertile males potentially carrying Cas9induced Cctra monoallelic mutations also in the germ line. However, no pupae and adult flies emerged from this set of injections. When Cas9-sgtraEx1 RNP alone targeting Cctra were delivered into $400 \mathrm{XX}$ embryos, few adults developed $(3 \% ; 12 / 400)$ (Table 1 , set n. 2). It is likely that the copresence of dsRNA+Cas9/sgRNA molecules could have a combined higher lethal effect for unclear reasons. Injections of dsRNA-Cctra alone resulted in 78 adults out of $200 \mathrm{XX}$ embryos injected, with $73 \mathrm{XX}$ being masculinized individuals and 5 being intersexes (Table 1, set n. 3). A similar survival rate was obtained when we injected sgtraEx1 RNA molecules but no effect $(1 \mu \mathrm{g} / \mu \mathrm{L})$, at a 5 times higher concentration being more susceptible to RNA degradation (70 females out of 160 injected embryos; Table 1, set n. 4).

Although upon Cas9-sgtraEx1 RNP injections we observed a very limited survival rate, the $\mathrm{XX}$ adults displayed some interesting features (Table 1, set n. 2). Indeed, the $G_{0}$ progeny was composed not only of six unaffected females, as we expected (Fig. 2 set 2), but also six XX males. These findings are suggestive of an unusually high rate of biallelic mutations. It is also worth noting that no intersexes were observed, suggesting an all-or-none effect on Cctra female-specific function of the Cas9 + sgtraEx1 injection.

Considering the apparently high efficiency of the ribonucleoprotein injections in set n. 2 (Table 1) in masculinizing $50 \%$ of the $\mathrm{G}_{0} \mathrm{XX}$ individuals, we reasoned that 1) also the $\mathrm{XX}$ females contained at least some somatic 
Table 1 XX-only embryonic injection sets

\begin{tabular}{|c|c|c|c|c|c|c|c|c|c|}
\hline $\begin{array}{l}\text { Injection } \\
\text { set }\end{array}$ & Cas9 delivery / [] & sgRNA name / [] & dsRNA name / [] & $\begin{array}{l}\text { XX } \\
\text { embryos }\end{array}$ & $\begin{array}{l}\text { XX } \\
\text { Larvae }\end{array}$ & $\begin{array}{l}\mathrm{XX} \\
\text { Females }\end{array}$ & $\begin{array}{l}\mathrm{XX} \\
\text { Males }\end{array}$ & $\begin{array}{l}\mathrm{XX} \\
\text { intersexes }\end{array}$ & $\begin{array}{l}\text { Cctra male- } \\
\text { specific in XX }\end{array}$ \\
\hline 1 & $\begin{array}{l}\text { Cas9 Protein / } \\
1,8 \mu \mathrm{g} / \mu \mathrm{L}\end{array}$ & $\begin{array}{l}\text { sgtraEx1 / } \\
200 \mathrm{ng} / \mu \mathrm{L}\end{array}$ & $\begin{array}{l}\text { dsRNA-Cctra / } \\
0.5 \mu \mathrm{g} / \mu \mathrm{L}\end{array}$ & 370 & 48 & 0 & 0 & 0 & - \\
\hline 2 & $\begin{array}{l}\text { Cas9 Protein / } \\
1,8 \mu \mathrm{g} / \mu \mathrm{L}\end{array}$ & $\begin{array}{l}\text { sgtraEx1 / } \\
200 \mathrm{ng} / \mu \mathrm{L}\end{array}$ & - & 400 & 60 & 6 & 6 & 0 & yes in $X X$ males \\
\hline 3 & - & - & $\begin{array}{l}\text { dsRNA-Cctra / } \\
0.5 \mu \mathrm{g} / \mu \mathrm{L}\end{array}$ & 200 & 83 & 0 & 73 & 5 & - \\
\hline 4 & - & $\begin{array}{l}\text { sgtraEx1 / } \\
1 \mu \mathrm{g} / \mu \mathrm{L}\end{array}$ & - & 160 & 75 & 70 & 0 & 0 & no \\
\hline 5 & $\begin{array}{l}\text { Plasmid-dCas9 / } \\
1 \mu \mathrm{g} / \mu \mathrm{L}\end{array}$ & $\begin{array}{l}\text { sgtraEx1 / } \\
1 \mu \mathrm{g} / \mu \mathrm{L}\end{array}$ & - & 290 & 37 & 7 & 0 & 3 & yes \\
\hline 6 & $\begin{array}{l}\text { Cas9 Protein / } \\
1,8 \mu \mathrm{g} / \mu \mathrm{L}\end{array}$ & $\begin{array}{l}\text { sgtraEx1 / } \\
200 \mathrm{ng} / \mu \mathrm{L}\end{array}$ & - & 40 & - & - & - & - & yes \\
\hline 7 & $\begin{array}{l}\text { Plasmid-Cas9 / } \\
1 \mu \mathrm{g} / \mu \mathrm{L}\end{array}$ & $\begin{array}{l}\text { sgtraEx1 / } \\
1 \mu \mathrm{g} / \mu \mathrm{L}\end{array}$ & - & 40 & - & - & - & - & yes \\
\hline 8 & $\begin{array}{l}\text { Plasmid-dCas9 / } \\
1 \mu \mathrm{g} / \mu \mathrm{L}\end{array}$ & $\begin{array}{l}\text { sgtraEx1 / } \\
1 \mu \mathrm{g} / \mu \mathrm{L}\end{array}$ & - & 40 & - & - & - & - & yes \\
\hline 9 & buffer & buffer & buffer & 40 & - & - & - & - & no \\
\hline 10 & - & $\begin{array}{l}\text { sgtraEx1 / } \\
1 \mu \mathrm{g} / \mu \mathrm{L}\end{array}$ & - & 40 & - & - & - & - & no \\
\hline 11 & $\begin{array}{l}\text { Cas9 Protein / } \\
1,8 \mu \mathrm{g} / \mathrm{\mu L}\end{array}$ & - & - & 40 & - & - & - & - & no \\
\hline 12 & $\begin{array}{l}\text { Plasmid-Cas9 / } \\
1 \mu \mathrm{g} / \mu \mathrm{L}\end{array}$ & - & - & 40 & - & - & - & - & no \\
\hline 13 & $\begin{array}{l}\text { Plasmid-dCas9 / } \\
1 \mu \mathrm{g} / \mu \mathrm{L}\end{array}$ & - & - & 40 & - & - & - & - & no \\
\hline
\end{tabular}

clones bearing monoallelic Cctra mutations, though with no phenotypic effect, and that 2) a high mutagenic rate could also be present in the germ lines of these XX female adults as in the XX males. Assuming a 20-50\% transmission rate of mutant alleles for each $G_{0}$ parent to the next progeny as previously observed in the Medfly [16], the probability to observe a double mutant individual in the $G_{1}$ progeny (Fig. 2) would be in a range of 4$25 \%$. When we crossed among them the six XX males and six XX females from injection set n. 2 (Table 1), all 100 individuals of $G_{1}$ were females, indicating that the six XX masculinized fathers were fertile. However, the absence of XX males in the $G_{1}$ progeny indicated that, if any Cctra mutation was induced by Cas 9 in the parental germ lines (male and female ones), the transmission rate was lower than $10 \%$ for each parent as the expected $G_{1}$ heteroallelic mutants frequency would be less than $1 \%$ of the progeny (hence not detectable among a number of 100 individuals).

Lack of indels in the targeted Cctra region in most cDNA clones from $X X G_{0}$ males and in all genomic DNA clones from $G_{0} X X$ females

The six females and six XX males, which composed the $G_{0}$ progeny of set $n$. 2, were analyzed respectively by
RT-PCR and genomic PCR, to investigate Cctra splicing and DNA sequence of the targeted site.

The six reverted $\mathrm{XX} \mathrm{G}_{0}$ males showed only malespecific Cctra transcripts, as expected for adult flies having a full fertile male phenotype (Fig. 3). These data, together with the absence of female-specific Cctra mRNAs in all six males, suggested the presence of biallelic mutations in most, if not all, of the somatic cells of these XX $\mathrm{G}_{0}$ males. Shotgun plasmid cloning of the RT-PCR reactions from the six $\mathrm{XX} \mathrm{G}_{0}$ males, followed by PCR colony screening of 30 clones (five colonies A-E, for each of the six XX males) led us to arbitrarily select 13 clones for sequencing (two or three cDNA clones for each male) (Additional file 1: Figure S2 and Figure S3)(Additional file 2). The splicing isoforms detected in the six XX males corresponded mostly to the know M1 and M2 Cctra male-specific isoforms. Out of 13 cDNA clones from the six XX males, seven correspond to malespecific Cctra isoform M1 (59 aa), two correspond to the male-specific isoform M2 (99 aa), one to a new splicing male-specific isoform (Male 1D encoding a 44 aa long protein isoform, named M3), one to the femalespecific isoform (429 aa) and one to an unspliced longer isoform (male 5C) (Additional file 1: Figure S3; Additional file 2) [6]. Very surprisingly, despite the observed Cctra male-specific full shift, 11 cDNA clones showed 


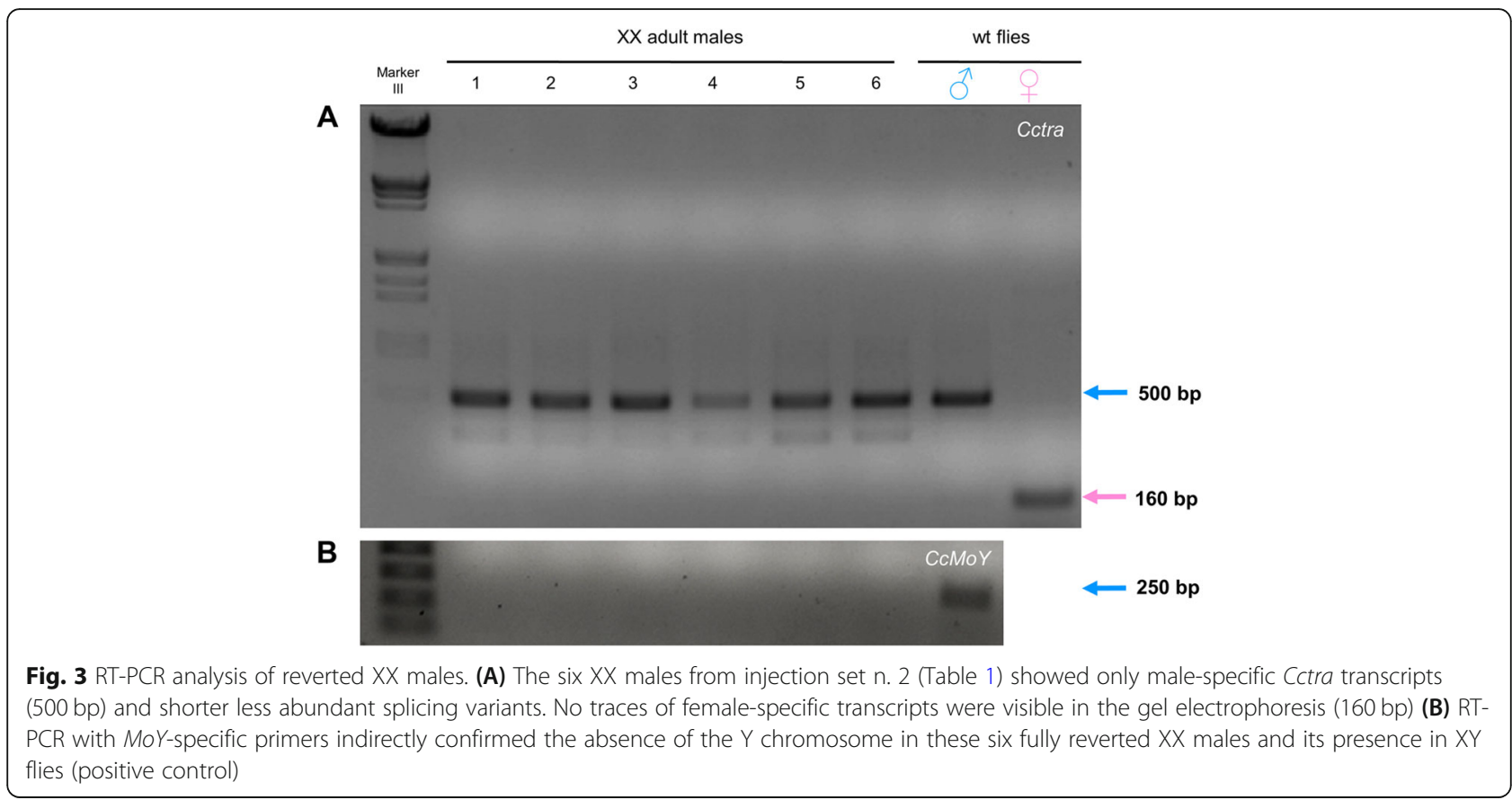

only wild-type sequences (Additional file 1: Figure S3). In one XX male, we have found a cDNA showing a 16 bp long deletion (cDNA 3E), in addition to a wild type cDNA clone (cDNA 3B) (Additional file 1: Figure S1D). The mutated cDNA encodes a 35 aa long CcTRA truncated protein. Another XX male contained two wild type (5B and 5D) cDNA clones and a mutated one lacking of $5 \mathrm{bp}$ (5C) (Additional file 1: Figure S1C). This cDNA Male $5 \mathrm{C}$ encoded a truncated CcTRA protein of only 42 aa (Additional file 1: Figure S1C). The male 5C cDNA from unspliced Cctra RNA seems to correspond to a previously described RNA only present in adult females [6]. The female specificity of this unspliced product could be due to the binding of female-specific CcTRA/ CcTRA-2 complex to the Cctra pre-mRNA required for the autoregulation. Interestingly, the Cctra unspliced isoform we found in one XX male contains also the Cas9induced $5 \mathrm{bp}$ deletion. We speculated that the $5 \mathrm{bp}$ long deleted region of Cctra could be involved in enhancing the recognition of the $5^{\prime}$ donor site, which is only $20 \mathrm{bp}$ downstream to the deletion by the spliceosome. We have found that these $5 \mathrm{bp}$ are perfectly conserved in other Tephritidae tra orthologues, suggesting their requirement for proper Cctra male-specific default splicing (Additional file 1: Figure S1E). On the other hand, the cDNA 3E containing even a larger deletion (16 bp) of the same Cctra region performed a male-specific splicing, indicating that this sequence ( $14 \mathrm{bp}$ out of $20 \mathrm{bp}$ targeted sequence conserved in 5 distantly related Tephritidae species; Additional file 1: Figure S1E) is not strictly required to perform this alternative splicing but could be involved in performing the female-specific one. Collectively, these data showed intriguingly that the six XX males had a full switch from female-specific to malespecific splicing of Cctra, even in the presence of a very low (hence, mostly monoallelic) or even zero number of mutant Cctra alleles.

As the six XX females showed a normal female phenotype, female-specific Cctra splicing was expected to be found, even if they were carrying monoallelic Cctra mutations (Fig. 2). Hence, Cctra genomic DNA (rather than RNA or cDNA) was analyzed and eight plasmid clones showed only Cctra wild-type sequences (Additional file 1: Figure S4; Additional file 3). The lack of Cctra mutations in the DNA clones from all six $G_{0}$ females and in most of the cDNA from the six $\mathrm{XX}$ males is consistent with the absence of $\mathrm{XX}$ mutant heteroallelic $\mathrm{Cctra}^{-1} / \mathrm{Cctra}^{-2}$ males we observed in their $\mathrm{G}_{1}$ progeny.

\section{Embryos co-injections of dCas9 and sgtraEx 1 lead to partially masculinized XX embryos and adult flies, indicating a long-lasting effect in the absence of mutations}

We speculated that this Cas9 ribonucleoprotein complex was able to bind but not efficiently cut the Cctra DNA target site. The dead Cas9 (dCas9) is a mutant Cas9 which lacks of only the endonuclease activity and it can be used to perform transient transcriptional repression, named CRISPR interference, or iCRISPR [23-25]. We co-injected the sgRNA sgtraEx1 $(1 \mu \mathrm{g} / \mu \mathrm{l})$ with a plasmid bearing a dCas9 transgene under a Drosophila actin promoter, expecting that dCas9 would be produced after 
transcription and translation and would bind the available sgRNA [24] (Table 1, set n. 5). A higher concentration of sgRNA $(1 \mu \mathrm{g} / \mu \mathrm{l}$; see mat. \& meth.) was used in the injected mix because we expected that these RNA molecules being not pre-assembled with Cas9 before injections, could have been more exposed to degradation. This high concentration of sgRNA alone had no effect on Cctra female-specific splicing in injected XX embryos and in developed XX adults (Table 1, set n. 10; Fig. 4, B). Three out of ten XX adult females showed malformations of the gonadal apparatus, which suggested a mild masculinization effect (Additional file 1: Figure S5A). RT-PCR analysis of Cctra on RNA from these three XX adult flies confirmed the presence of also male-specific RNAs, indicating that the ovipositor malformations are likely the result of a partial masculinization during development (Additional file 1: Figure S5B). These data indicated that the dCas9 can induce a partial masculinization of $\mathrm{XX}$ embryos and a stable shift toward male-specific splicing of Cctra likely in some somatic clones.

We investigated the sex-specific splicing pattern of Cctra in XX-only embryos, injected at $1 \mathrm{~h}$ after egg laying, and developed for additional $14 \mathrm{~h}$ (set n. 6-13). Injections of Cas9 recombinant protein+sgtraEx1 RNP (set n. 6), or co-injections of sgtraEx1 with plasmids encoding either Cas9 (driven by E1 early promoter [23]) or dCas9 (set $n .7$ and set n. 8) led to the appearance of additional malespecific Cctra transcripts (Table 1, Fig. 4A). Injections of only buffer, sgtraEx1, Cas9 protein, Cas9-encoding and dCas9-encoding plasmids had no effect (Fig. 4B). These data suggest that, as Cas9, also dCas9 can induce malespecific splicing of Cctra during the first hours of development. Hence, no Cctra mutations are required for this splicing change.

\section{Discussion}

The Cas9-sgtraEx1 RNPs injected into XX Medfly embryos led to a masculinization of 50\% of XX individuals. All six XX males showed by RT-PCR and cDNA sequencing analyses mostly male-specific Cctra isoforms, concordant with the observed male phenotype. Unexpectedly, no indel mutations were detected in cDNA fragments from the Cas9 targeted site in four out of six males. Two XX males showed a mix of wild type and mutated cDNA fragments, suggesting the presence of monoallelic mutations in some cellular regions. XX males and XX females from injected embryos produced a $G_{1}$ consisting of only XX females, which indicated absence of homozygous individuals for mutant Cctra alleles. We concluded that very low mutation rate, if any, was reached not only in the soma but also in the germ line of injected $G_{0}$ individuals. In contrast, Meccariello et al. [16], observed that mutant mosaic $G_{0}$ flies

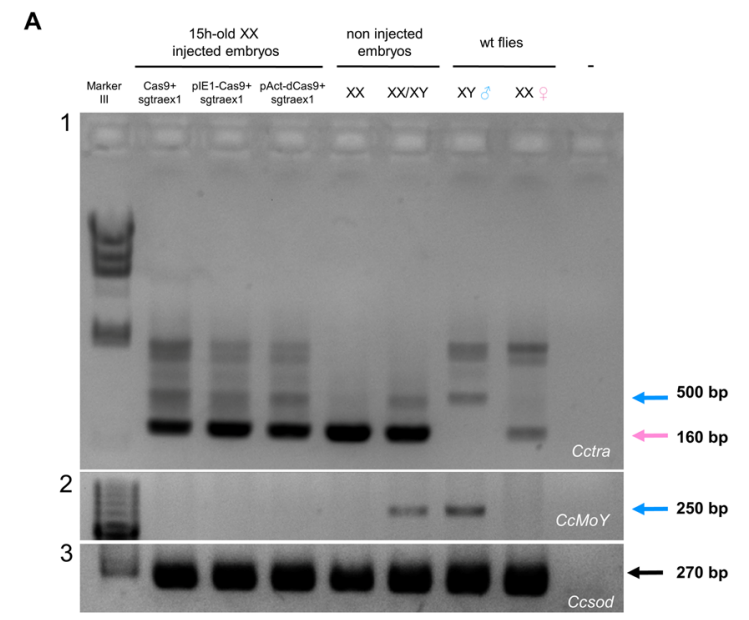

B

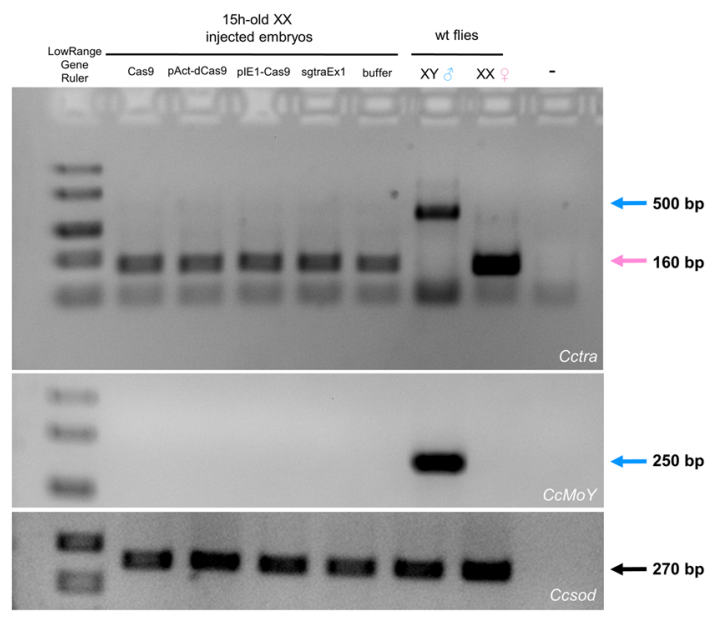

Fig. 4 Molecular partial masculinization of XX embryos by dCas9 targeting Cctra. (A) RT-PCR analysis of Cctra sex-specific transcripts in $15 \mathrm{~h}$ old XX embryos, following injections of various samples (Table 1 , set $n .6-8$ ) at $1 \mathrm{~h}$ after egg laying (Panel 1). The XX embryos were injected respectively with Cas9 protein+sgtraEx1 (first lane), with sgtraEx1 + Cas9-encoding plasmid (plE1-Cas9; second lane) and with dCas9-encoding plasmid (pAct-dCas9) (third lane). Male-specific Cctra transcripts were detected (500 bp cDNA fragment) in all three samples of injected XX embryos, in addition to the female-specific transcripts (160 bp cDNA fragment). RT-PCR analysis of the Y-linked MoY gene indirectly confirmed the absence of $Y$ chromosome in all 3 samples and in $X X$ females, and its presence in a mixed $X X / X Y$ embryos sample and in adult $X Y$ flies (Panel 2). CCSOD positive control is shown in panel 3. (B) Negative controls. RT-PCR of Cctra sex-specific transcripts in $15 \mathrm{~h}$ old $\mathrm{XX}$ embryos, following injections at $1 \mathrm{~h}$ after egg laying (Table 1, set $\mathrm{n}$. 9-13). The XX embryos were injected respectively with Cas9 protein, Cas9-encoding plasmid, dCas9-encoding plasmid, with sgtraEx1 and with buffer alone. Only female-specific Cctra transcripts were detected (160 bp) in all samples of injected XX embryos (Panel 1). In Panel 2 and 3, the control of the karyotype and positive controls, conducted as in A, are shown 
mutagenized by Cas9 in the white-eye gene transmitted germ line mutations to the progeny at a very high rate (up to $100 \%$ ). Hence, the Cas9-sgtraEx1 is likely very inefficient in mediating Cas9 gene disruption but surprisingly very efficient in masculinizing $\mathrm{XX}$ individuals. These data suggested that the Cas9 ribonucleoprotein promoted masculinization by inducing male-specific Cctra splicing in 50\% of XX embryos which developed into adults by a mechanism different than gene disruption.

We reasoned that the sgRNA designed for the CRISPR experiment and/or the Cctra targeted region structural features posed limitations in the second step of the Cas9 action, namely the endonuclease activity, but not in the first step, the binding to the Cctra genetic locus. Indeed, a two-state model for Cas9 binding and cleavage was recently proposed: a seed match triggers binding but only extensive pairing with target DNA leads to cleavage [26]. Target sequence mismatches can induce the Cas9-RNP complex to bind also off-target sites without DNA cleavage, because the transition to the active conformation is prevented [26, 27]. Co-injections of sgtraEx1 and a plasmid encoding dCas9 into XX embryos induced a partial shift of Cctra splicing toward male-specific pattern after few hours of development and the development of XX females (three out of ten) with malformed ovipositor. These females were found to be molecular intersexes, as they showed both female-specific and male-specific Cctra isoforms. Also, these malformed females showed a mix of male and female-specific Cctra transcripts and, hence, correspond to partially masculinized individuals (intersexes) [6]. Similar malformed ovipositors were observed by Pane et al. [6] following embryonic RNA against Cctra. The lack of fully masculinized XX individuals in the progeny of ten individuals suggests a reduced efficiency of dCas9 in masculinizing XX individuals. However, this could be due to the different delivery methods of Cas9 and dCas9. In the first case, purified recombinant Cas9 ready to act was injected, while in the second case a dCas9 encoding transgene was transcribed from a Drosophila actin promoter, after embryos injections of a plasmid. Lower efficiency is to be expected in the second delivery method, due to the transcription and translation steps required to express dCas9. The transient binding of Cas9 RNPs to the 5' Cctra DNA region could have reduced the Cctra zygotic transcription during the first hours of embryogenesis and, hence, the accumulation of female specifically spliced Cctra mRNA, promoted by the maternal Cctra contribution in XX embryos. This transient reduction of newly transcribed Cctra female-specific mRNA and of the encoded CcTRA protein in the XX embryos could have blocked the establishment of Cctra female-specific autoregulation leading to a negative epigenetic effect on Cctra. Similarly, a transient depletion of Cctra mRNA by embryonic RNAi led to a collapse of its positive autoregulation and to obtain XX fertile males expressing male-specific Cctra RNAs [6].

\section{Conclusions}

The question if CcTRA male-specific ORFs are required for male-specific Cctra splicing remains still open, as biallelic Cctra mutant XX males were not obtained. The second question if the chosen sgtraEx1 guide RNA is suitable for future gene drive strategy aimed to efficient mutagenesis by Cas 9 had a conclusive and negative reply, as very low mutagenesis rate was observed. However, these data support the hypothesis that the transient binding of the Cas9-sgtraEx1 ribonucleoprotein complex on the first Cctra exon during the first hours of embryogenesis led to a repression of the establishment of the Cctra female-specific autoregulation in XX embryos even in the absence of induced mutations. Our study raises new general issues concerning the use of CRISPR/ Cas9 method. We serendipitously uncovered a novel problem of unplanned stable changes in the expression of genes able to autoregulate, which calls for further investigation. If a Cas9+sgRNA binds to off target sequences of autoregulating bistable genes, this event can provoke long lasting epigenetic effects even in the absence of DNA mutations.

\section{Methods}

\section{Rearing of Ceratitis capitata}

Wild type (WT) and transgenic Medfly lines were maintained under standard rearing conditions. The WT Benakeion strain, which has been reared in laboratories for more than 20 years, was obtained from P. A. Mourikis 30 years ago (Benakeion Institute of Phytopathology, Athens, Greece). The strains were reared in standard laboratory conditions at $25{ }^{\circ} \mathrm{C}, 70 \%$ relative humidity and $12: 12 \mathrm{~h}$ light-dark regimen. Adult flies were fed yeast/ sucrose powder (1:2).

\section{RNP complex assembly and injections}

Cas9 was expressed as his-tagged protein and purified from bacteria [16, 19]. sgRNA was designed using the CHOPCHOP online software [28]. The lack of SNPs within this $20 \mathrm{nt}$ long sequence in three different Ceratitis lines (Benakeion, used in this study, ISPRA [22] and FAM18 Ceratitis [8]) suggested that no Cas9-resistant Cctra alleles would be already present in individuals of these lab strains. Template for sgRNA in vitro transcription were generated by annealing two complementary oligonucleotides (PAGE-purified, Life Technologies) as previously described $[16,21]$, using the primers $\mathrm{F}$ sgtraEx1 and Reverse-Crispr from Life Technologies (Additional file 4). sgRNA was synthesized according to 
instructions of the Megascript $^{\circ} \mathrm{T} 7 \mathrm{kit}$ (Ambion) with $1 \mu \mathrm{g}$ of DNA template and a $5^{\prime}$ flanking T7 promoter. After RNA synthesis, the template was removed by incubating with TurboDNase (Ambion) for $15 \mathrm{~min}$ at $37^{\circ}$. Prior to the injection, the RNP complex was prepared by mixing $1.8 \mu \mathrm{g} / \mu \mathrm{L}$ of purified Cas9 protein with approximately $200 \mathrm{ng} / \mu \mathrm{L}$ of sgtraEx1, containing $300 \mathrm{mM} \mathrm{KCl}$ [16]. The mix was incubated for $10 \mathrm{~min}$ at $37^{\circ} \mathrm{C}$. A glass needle was filled with the pre-loaded sgtraEx1-Cas9 mix and the injection was performed into the posterior end of embryos collected $45 \mathrm{~min}$ after egg laying as described for RNA interference in Ceratitis capitata [6]. When injecting sgRNA alone, a 5 times higher concentration $(1 \mu \mathrm{g} / \mu \mathrm{L})$ was used in the injected mix, because we expected that these RNA molecules, being not preassembled with Cas9 before injections, could have been more exposed to degradation.

\section{RNAi and XX-only progeny production}

A Cctra cDNA 800 bp long fragment was PCR amplified using RNA from female adults of $C$. capitata and longer 164+/900- primers, introducing a T7 promoter sequence at each extremity. In vitro transcription of Cctra dsRNA was performed using the Ambion MEGAscript ${ }^{\circ}$ RNAi kit T7 RNA polymerase, following manufacturer instructions. Embryonic RNAi $(0.5 \mu \mathrm{g} / \mu \mathrm{L}$ dsRNA solution) was used to repress Cctra in XX/XY embryos and to produce male only progeny. Single males from this progeny were crossed with three females in small cages and the crosses having XX males were identify by Y-specific PCR (Y-specific primers) [8] on a small sample of laid embryos.

\section{dCas9 encoding plasmid and injections}

Plasmid expressing dCas9 under the control of the Drosophila melanogaster actin promoter was kindly provided to GS by Lenny Rabinow (Perrimon's lab, Harvard, USA) [23]. A mix containing $1 \mu \mathrm{g} / \mu \mathrm{L}$ of pAct-dCas 9 plasmid and $1 \mu \mathrm{g} / \mu \mathrm{L}$ of sgtraEx 1 transcribed in vitro into the posterior end of embryos. We used 5 times higher concentration of sgRNA because we expected that these small RNA molecules being not pre-assembled with Cas9 before injections, could have been more exposed to degradation.

\section{RNA extraction and RT-PCR}

Total RNA was extracted from pools of embryos, intersexes, male and female adults using TRIzol $^{\circ}$ Reagent (Invitrogen ${ }^{\circ}$ ) following manufacturer instructions. Oligo-dT-primed cDNA was prepared from DNAse-treated total RNA using EuroScript ${ }^{\circ} \mathrm{m}$ MLV reverse transcriptase (Euroclone ${ }^{\circ}$ ). RT-PCR expression analysis was performed with the following primers: Cctra 164+/320-, CcSOD+/CcSOD- and CcMoY A+/CcMoY A- (Additional file 4). For XX malformed females obtained from XX only embryos with dCas9 and sgtraEx1 Cctra RT-PCR was performed using Cctra 164+/900- primers pair, annealing on the same exon as Cctra 320- primer (Additional file 4). Gel electrophoresis diagnostic amplicon run was performed using Marker III (Lambda genomic DNA digested with EcoRI/HindIII) or $100 \mathrm{bp}$ ladder from Thermo Scientific ${ }^{\circ}$.

\section{DNA extraction and molecular analysis}

DNA extraction was performed, with minor modifications, according to the protocol of Holmes and Bonner et al. [29]. Adult XX female flies $G_{0}$ were placed in a 1.5 $\mathrm{ml}$ tube and manually crushed with a pestle in $200 \mathrm{ml}$ Holmes Bonner buffer (Urea 7 M, 281 Tris- $\mathrm{HCl} 100 \mathrm{mM}$ pH 8.0, EDTA $10 \mathrm{mM}$ pH 8.0, NaCl $350 \mathrm{mM}$, SDS 2\%). Subsequently, DNA was purified by phenol/chloroform extraction, followed by chloroform extraction and ethanol precipitation. The pellet was resuspended in $30 \mu \mathrm{l}$ water containing RNase A.

\section{cDNA and gDNA cloning and sequencing}

PCR cDNA fragments from XX adult males were cloned into pGEM-T Easy Promega ${ }^{\circ}$ vector according manufacturer instruction. PCR colony screening was carried out using $164+$ and 320 - primers. Positive colonies were used to extract plasmid DNA which was sequenced using Applied Biosystem ${ }^{\circ}$ Big Dye v 3.1. Genomic DNA from the six $G_{0} X X$ females was used as template to amplify the region encompassing the target sites, using the primers Cctra 164+ and Cctra 164-Rev (Additional file 4). DreamTaq (Life Technologies) polymerase was used for PCR amplifications according to the manufacturer's instructions. The PCR products were purified with StrataPrep PCR Purification Kit (Agilent Technologies) and subcloned using StrataClone PCR cloning Kit (Agilent Technologies). Positive clones were sequenced by Sanger method and ABI 310 Automated Sequencer (Applied Biosystems) using the primer Cctra 164+ (Additional file 4).

\section{RNA extraction from injected embryos after $15 \mathrm{~h}$ of development}

The pools of 40 embryos injected with various mixes (Table 1) were let develop for $15 \mathrm{~h}$ at $25^{\circ} \mathrm{C}, 70 \%$ relative humidity. The embryos were then detached from the cover slip using heptane, which dissolves the glue, and collected in a $1.5 \mathrm{~mL}$ tube. They were then washed three times with 1X PBS to remove heptane before RNA extraction was performed using TRIzol $^{\circ}$ Reagent (Invitrogen $^{\circ}$ ) following manufacturer instructions. The RNA samples were analyzed for Cctra splicing pattern by RTPCR using the primers Cctra 164+ and Cctra 900(Additional file 4).). The cDNA and genomic sequences 
were deposited at the GenBank database with the following accession numbers: MW200161 to MW200180.

\section{Supplementary Information}

The online version contains supplementary material available at https://doi. org/10.1186/s12863-020-00941-4.

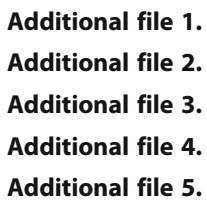

\section{Abbreviations}

SIT: Sterile Insect Technique; ORF: Open Reading Frame; CRISPR: Clustered Regulated InterSpaced Palindromic Repeats; RNP: Ribo-Nucleo-Proteic Complex; dsRNA: double strand RNA; sgRNA: single guide RNA; iCRISPR: interference CRISPR; dCas9: dead Cas9; SNP: Single Nucleotide Polymorphism; RNAi: RNA interference; WT: Wild-Type

\section{Acknowledgements}

We would like to thank Lenny Rabinow (Harvard University, USA) for providing dCas9 plasmid vector and Donald L. Jarvis (University of Wyoming, USA) for providing E1-P-Cas9. GSA and AM initiated the project. We wish to thank Luigi Vitagliano (IBB-CNR, Italy) for in depth discussion, insights and manuscript reviewing. This study was benefited from discussions at International Atomic Energy Agency funded meetings for the Coordinated Research Project "Comparing Rearing Efficiency and Competitiveness of Sterile Male Strains Produced by Genetic, Transgenic or Symbiont-based Technologies". We thank the anonymous reviewers and the guest editor for their helpful and generous suggestions to improve the description of this study.

\section{About this supplement}

This article has been published as part of BMC Genetics Volume 21 Supplement 2, 2020: Comparing rearing efficiency and competitiveness of sterile male strains produced by genetic, transgenic or symbiont-based technologies. The full contents of the supplement are available online at https://bmcgenet. biomedcentral.com/articles/supplements/volume-21-supplement-2.

\section{Authors' contributions}

AM, AG, MGl, GDC, performed set n. 1-4 embryonic injections (Table 1) and the embryonic injections of Cctra dsRNA+Cas9 + sgtraEx1, which led to $100 \%$ lethality. PP performed PCR, cloning and sequencing analyses of the 6 XX males and 6 XX females from of injection set $n .2$ (Table 1). PP and GSO performed injection set n. 5 and RT-PCR of the $3 X X$ malformed females. PP and GV performed injection set n. 6-9 and GV performed RT-PCR analyses on the 15 h-old injected embryos. PP performed the cross of $G_{0}$ males and females from set $n .2$ and visually screened the $G_{1}$ progeny. AM, PP, AG, MGI, GDC, GSO and GV maintained the Medfly strains, preparing larval and adult food, performing crosses and collecting embryos. GSA wrote the paper, with the inputs of MDR, SA, MS, and AM. GS prepared the figures, with contributions of AM, MS and PP. All authors contributed with minor corrections/editing of the manuscript, especially AG. All the authors have read and approved the final version of the manuscript.

\section{Funding}

The PhD Biology program at University of Naples Federico II supported this study with fellowships to AM and PP. The Department of Biology supported this study with small-budget grants (Ricerca Dipartimentale) to GSA, SA and MS, for data collection and their interpretation. MDR acknowledges support from the UZH University Research Priority Program Evolution in Action. Publication costs are funded by the Joint FAO/IAEA Division of Nuclear Techniques in Food and Agriculture, IAEA (CRP No.: D4.20.16) Vienna, Austria. The funding bodies provided the financial means to allow the authors to carry out the study and the funding bodies played no role in the design of the study and collection, analysis, and interpretation of data and in writing the manuscript.

\section{Availability of data and materials}

The cDNA and genomic sequences were deposited at the GenBank database with the following accession numbers: MW200161 to MW200180.

Ethics approval and consent to participate

Not applicable.

\section{Consent for publication}

Not applicable.

\section{Competing interests}

The authors declare absence of competing interests.

\section{Author details}

${ }^{1}$ Department of Biology, University of Naples Federico II, 80126 Naples, Italy. ${ }^{2}$ Department of Molecular Life Sciences and SIB Swiss Institute of

Bioinformatics, University of Zurich, Winterthurerstrasse 190, CH-8057 Zurich, Switzerland

Published: 18 December 2020

\section{References}

1. De Meyer M, Robertson MP, Peterson AT, Mansell MW. Ecological niches and potential geographical distributions of Mediterranean fruit Fly (Ceratitis capitata) and Natal fruit Fly (Ceratitis rosa). J Biogeogr. 2008;35(2):270-81.

2. Franz G. Genetic Sexing Strains in Mediterranean Fruit Fly, an Example for Other Species Amenable to Large-Scale Rearing for the Sterile Insect Technique. In: Dyck VA, Hendrichs J, Robinson A, editors. Sterile Insect Technique. Dordrecht: Springer; 2005. https://doi.org/10.1007/1-4020-4051-2_16.

3. Rendon P, Mclnnis D, Lance D, Stewart J. Medfly (Diptera: Tephritidae) genetic sexing: large-scale field comparison of males-only and bisexual sterile fly releases in Guatemala. J Econ Entomol. 2004;97:1547-53.

4. Lutrat C, Giesbrecht D, Marois E, Whyard S, Baldet T, Bouyer J. Sex Sorting for Pest Control: It's raining men! Trends Parasitol. 2019;35(8):649-62. https://doi.org/10.1016/j.pt.2019.06.001 Epub 2019 Jun 26.

5. Saccone G, Pane A, De Simone M, Salvemini M, Milano A, Annunziata L, Mauro U, Polito LC. New Sexing Strains for Mediterranean Fruit Fly Ceratitis capitata: Transforming Females into Males. In: MJB V, Robinson AS, Hendrichs J, editors. Area-Wide Control of Insect Pests. Dordrecht: Springer; 2007.

6. Pane A, Salvemini M, Delli Bovi P, Polito C, Saccone G. The transformer gene in Ceratitis capitata provides a genetic basis for selecting and remembering the sexual fate. Development. 2002;129:3715-25. Development (Cambridge, England). 129. 3715-25. https://doi.org/10.3410/f.1001642.116658.

7. Tian M. Maniatis TA splicing enhancer exhibits both constitutive and regulated activities. Genes Dev. 1994;8(14):1703-12.

8. Meccariello A, Salvemini M, Primo P, Hall B, Koskinioti P, Dalíková M, Gravina A, Gucciardino MA, Forlenza F, Gregoriou ME, Ippolito D, Monti SM, Petrella V, Perrotta MM, Schmeing S, Ruggiero A, Scolari F, Giordano E, Tsoumani KT, Marec F, Windbichler N, Nagaraju J, Arunkumar K, Bourtzis K, Mathiopoulos KD, Ragoussis J, Vitagliano L, Tu Z, Papathanos PA, Robinson MD, Saccone G. Maleness-on-the-Y (MoY) orchestrates male sex determination in major agricultural fruit fly pests. Science. 2019;365(6460):1457-60. https://doi.org/ 10.1126/science.aax1318.

9. Saccone G, Salvemini M, Polito LC. The transformer gene of Ceratitis capitata: a paradigm for a conserved epigenetic master regulator of sex determination in insects. Genetica. 2011;139:99-111.

10. Bopp D, Saccone G, Beye M. Sex determination in insects: variations on a common theme. Sex Dev. 2014;8(1-3):20-8.

11. Vargas RI, Piñero JC, Leblanc L. An overview of pest species of Bactrocera fruit flies (diptera: Tephritidae) and the integration of biopesticides with other biological approaches for their management with a focus on the Pacific region. Insects. 2015;6(2):297-318. https://doi.org/10.3390/ insects6020297 Review.

12. Ekesi S, De Meyer M, Mohamed SA, Virgilio M, Borgemeister C. Taxonomy, ecology, and Management of Native and Exotic Fruit fly Species in Africa. Annu Rev Entomol. 2016;61:219-38.

13. Nagaraju J, Saccone G. How sex is determined in insects? Preface. J Genet. 2010;89(3):269-70.

14. Handler AM. Prospects for using genetic transformation for improved SIT and new biocontrol methods. Genetica. 2002;116(1):137-49 Review. 
15. Louis C, Savakis C, Kafatos FC. Possibilities For Genetic Engineering In Insects Of Economic Interest, Elsevier 1986, II. In: Tern Symp Fruit Flies/Crete Sept; 1986.

16. Meccariello A, Monti SM, Romanelli A, Colonna R, Primo P, Inghilterra MG, Del Corsano G, Ramaglia A, lazzetti G, Chiarhours A, Patti F, Heinze SD, Salvemini M, Lindsay H, Chiavacci E, Burger A, Robinson MD, Mosimann C, Bopp D, Saccone G. Highly efficient DNA-free gene disruption in the agricultural pest Ceratitis capitata by CRISPR-Cas9 ribonucleoprotein complexes. Sci Rep. 2017;7(1):10061.

17. Saccone G, Peluso I, Artiaco D, Giordano E, Bopp D, Polito LC. The Ceratitis capitata homologue of the Drosophila sex-determining gene Sex-lethal is structurally conserved, but not sex-specifically regulated. Development. 1998;125(8):1495.

18. Saccone G, Salvemini M, Pane A, Polito LC. Masculinization of XX Drosophila transgenic flies expressing Ceratitis capitata DSXM isoform. Inter Journal of Dev Biol. 2008:52(8):1051-7.

19. Salvemini M, Robertson M, Aronson B, Atkinson P, Polito C, Saccone G. Ceratitis capitata transformer-2 gene is required to establish and maintain the autoregulation of Cctra, the master gene for female sex determination. Int J Dev Biol. 2009;53:109-20. https://doi.org/10.1387/ijdb.082681ms.

20. Nagoshi RN, McKeown M, Burtis KC, Belote JM, Baker BS. The control of alternative splicing at genes regulating sexual differentiation in $D$. melanogaster. Cell. 1988 Apr 22;53(2):229-36.

21. Lee JS, Kwak SJ, Kim J, Kim AK, Noh HM, Kim JS, Yu K. RNA-Guided Genome Editing in Drosophila with the Purified Cas9 Protein. G3-Genes Genom Genet. 2014:4:1291-5.

22. Papanicolaou A, Schetelig MF, Arensburger P, Atkinson PW, Benoit JB, Bourtzis K, Castañera P, Cavanaugh JP, Chao H, Childers C, Curril I, Dinh H, Doddapaneni H, Dolan A, Dugan S, Friedrich M, Gasperi G, Geib S, Georgakilas G, Gibbs RA, Giers SD, Gomulski LM, González-Guzmán M, Guillem-Amat A, Han Y, Hatzigeorgiou AG, Hernández-Crespo P, Hughes DST, Jones JW, Karagkouni D, Koskinioti P, Lee SL, Malacrida AR, Manni M, Mathiopoulos K, Meccariello A, Murali SC, Murphy TD, Muzny DM, Oberhofer G, Ortego F, Paraskevopoulou MD, Poelchau M, Qu J, Reczko M, Robertson HM, Rosendale AJ, Rosselot AE, Saccone G, Salvemini M, Savini G, Schreiner P, Scolari F, Siciliano P, Sim SB, Tsiamis G, Ureña E, Vlachos IS, Werren JH, Wimmer EA, Worley KC, Zacharopoulou A, Richards S, Handler AM. The whole genome sequence of the Mediterranean fruit fly, Ceratitis capitata (Wiedemann), reveals insights into the biology and adaptive evolution of a highly invasive pest species. Genome Biol. 2016;17:192.

23. Lin S, Ewen-Campen B, Ni X, Housden BE, Perrimon N. In vivo transcriptional activation using CRISPR/Cas9 in Drosophila. Genetics. 2015;201(2):433-42.

24. Mabashi-Asazuma H, Jarvis DL. CRISPR-Cas9 vectors for genome editing and host engineering in the baculovirus-insect cell system. Proc Natl Acad Sci. 2017:114(34):9068-73.

25. Qi LS, Larson MH, Gilbert LA, Doudna JA, Weissman JS, Arkin AP, Lim WA. Repurposing CRISPR as an RNA-guided platform for sequence-specific control of gene expression. Cell. 2013;152:1173-83.

26. Dagdas YS, Chen JS, Sternberg SH, Doudna JA, Yildiz A. A conformational checkpoint between DNA binding and cleavage by CRISPR-Cas9. Sci Adv. 2017;3(8):eaao0027.

27. Zhang L, Rube HT, Bussemaker HJ, Pufall MA. The effect of sequence mismatches on binding affinity and endonuclease activity are decoupled throughout the Cas9 binding site. bioRxiv. 2017:176255. https://doi.org/10. 1101/176255.

28. Montague TG, Cruz JM, Gagnon JA, Church GM, Valen E. CHOPCHOP: a CRIS PR/Cas9 and TALEN web tool forgenome editing. Nucleic Acids Res. 2014; 42:401-7.

29. Holmes DS, Bonner J. Preparation, molecular weight, base composition, and secondary structure of giant nuclear ribonucleic acid. Biochemistry. 1973; 12(12):2330-8.

\section{Publisher's Note}

Springer Nature remains neutral with regard to jurisdictional claims in published maps and institutional affiliations.

Ready to submit your research? Choose BMC and benefit from:

- fast, convenient online submission

- thorough peer review by experienced researchers in your field

- rapid publication on acceptance

- support for research data, including large and complex data types

- gold Open Access which fosters wider collaboration and increased citations

- maximum visibility for your research: over $100 \mathrm{M}$ website views per year

At BMC, research is always in progress.

Learn more biomedcentral.com/submissions 